\title{
Double crush syndrome: clinical review of an unsolved puzzle
}

\author{
Deven R. Kuruwa*, Easwar Elango, Kunal A. Shah
}

Department of Orthopaedics, Lokmanya Tilak Municipal Medical College \& General Hospital, Sion. Mumbai, India.

Received: 31 July 2020

Accepted: 11 September 2020

\section{*Correspondence:}

Dr. Deven R. Kuruwa,

E-mail: generalkuruwa@gmail.com

Copyright: (C) the author(s), publisher and licensee Medip Academy. This is an open-access article distributed under the terms of the Creative Commons Attribution Non-Commercial License, which permits unrestricted non-commercial use, distribution, and reproduction in any medium, provided the original work is properly cited.

\begin{abstract}
Double crush syndrome (DCS) involves compression of a peripheral nerve at two different segments. Median nerve is most commonly involved with proximal compression at the level of cervical spine and distal compression in the carpal tunnel. Little consensus exists in literature regarding its epidemiology, risk factors, pathophysiology and definitive treatment. The purpose of this article is to summarize our current knowledge about this disease process as well as to touch upon the controversies that have been generated in recent times.
\end{abstract}

Keywords: Double crush syndrome, Carpal tunnel syndrome, Cervical radiculopathy, Median nerve, Controversy

\section{INTRODUCTION}

Associated proximal symptoms like back, neck, shoulder and arm pain as well as failure of response to surgical decompression of median nerve in Carpal tunnel syndrome (CTS) lead to the hypothesis of Double crush syndrome (DCS).

DCS is due to the presence of compression at two sites along the path of a peripheral nerve. This hypothesis was first formulated by Upton in 1973 and further substantiated by Massey in 1981.,2

They postulated that compression of a peripheral nerve at one site increased its susceptibility to injury at another as a result of impaired bidirectional transport of essential nutrients across the axon. The degree of neuronal dysfunction caused by double crush has been shown to exceed the additive injury caused by individual compressions. ${ }^{3}$

Median nerve involvement in CTS and its association with Cervical radiculopathy $(\mathrm{CR})$ is the most widely studied example of DCS in literature. ${ }^{4,5}$

\section{EPIDEMIOLOGY}

CTS accounts for $90 \%$ of all upper limb entrapment neuropathies. ${ }^{6}$ Its incidence in the general population is 3$4 \%$ with $5: 1$ female to male ratio. ${ }^{7}$ It is more prevalent in adults between the age of 40 and 60 years. Incidence of CR in the general population is $<1 \%$ with male dominance and peak in the 6 th decade. ${ }^{8}$ Variable reports on co-existence of CR in patients with CTS exist, ranging from $6.7 \%$ to as high as $75.4 \%{ }^{9,10}$ Conflicting studies have failed to prove whether DCS is more prevalent in males or females. ${ }^{11,12}$

\section{RISK FACTORS}

The risk factors for CTS include occupation, repetitive hand movements, pregnancy, obesity, alcohol abuse and a plethora of medical conditions like diabetes mellitus, rheumatoid arthritis, amyloidosis, hypothyroidism, gout, tumors, infectious synovitis and other inflammatory pathologies. The risk factors for CR include age, smoking, obesity, poor sedentary lifestyle, osteoporosis and repetitive mechanical loading. Although the risk factors for these individual processes could lead to the causation of DCS, this seldom holds true. ${ }^{13}$ No consensus exists 
regarding the risk factors, epidemiology or pathophysiology of DCS in literature.

\section{PATHOPHYSIOLOGY}

The double crush hypothesis originally described by Upton states that axons that have been compressed at one site become susceptible to damage at another site, because of impaired axonal flow. ${ }^{1}$ Since then the exact mechanisms responsible for producing DCS have been a subject of controversy. In 2011, a panel of 17 international experts identified 14 possible mechanisms out of which 4 were highly plausible (table 1$){ }^{14}$

\section{PHYSICAL EXAMINATION}

CTS presents with pain, hypoesthesia and paresthesia in radial $3 \frac{1}{2}$ digits along the distribution of the median nerve. Thenar wasting may be seen in some cases. Phalen's test, Tinel's test and Durkan's compression test are performed to screen for the presence of CTS. In Phalen's test, wrist is kept in volar flexion for 60 seconds against gravity. In Durkan's test, thumb pressure is applied over the carpal tunnel for 30 seconds. In Tinel's test, median nerve is tapped over the carpal tunnel. These tests are considered positive if CTS symptoms are reproduced in the distribution of median nerve. Two-point discrimination, vibration and monofilament testing are additional tests which reproduce the sensory symptoms in CTS. The Katz hand diagram is a useful self-assessment test but with low sensitivity $(64 \%)$ and low specificity $(73 \%) .{ }^{15}$ In addition to the CTS symptoms, patients with DCS experience pain in the neck, upper back, shoulder or upper limb hypoesthesia ipsilaterally.

\section{DIAGNOSTIC TESTS}

When history and physical examination findings suggest DCS, additional tests are required to prove its existence. There are no established diagnostic criteria for DCS in literature.

Amongst the two electrodiagnostic studies, Nerve conduction study (NCS) is better than Electromyography (EMG) attributed to focal demyelination in CTS. Median nerve conduction study is the gold standard in the diagnosis of CTS with a specificity of $95-99 \% .{ }^{16}$ EMG is performed only if cervical disc herniation, brachial plexopathy or ulnar neuropathy are suspected (evaluating involvement of C8 and T1). Neuromuscular ultrasound is emerging as a valuable tool in addition to electrodiagnostic studies for CTS since the latter tends to underdiagnose 13 $27 \%$ cases. $^{17}$

NCS comprises of sensory conduction study and motor conduction study. The key finding for CTS is the slowing of conduction velocity in the median nerve $(<52 \mathrm{~m} / \mathrm{sec})$ localized to the carpal tunnel in sensory study. The peak latency of median Nerve sensory action potential (SNAP) is a reliable and popular parameter used for grading the severity of CTS. Motor nerve conduction is generally recorded over the Abductor pollicis brevis (ABP) muscle. Delay in its motor terminal latency $(>4.5 \mathrm{~ms})$ supports the diagnosis of CTS. NCS is more anomalous in DCS than in simple CTS, which supports the double crush hypothesis. ${ }^{18}$

Plain radiographs or Computed tomography (CT) scans may show loss of disc height and/or osteophyte formation in cervical radiculopathy. Magnetic resonance imaging (MRI) is the investigation of choice. It gives evidence of nerve root compression due to herniated disc or foraminal stenosis.

\section{MANAGEMENT}

Detection and treatment of all underlying pathological conditions like diabetes should be prioritized. Initial conservative measures include rest, avoidance of triggering movements, pharmacological therapy with oral steroids or Non-steroidal anti-inflammatory drugs (NSAIDS), short duration of immobilization in wrist splint with or without a cervical collar, therapeutic ultrasound and graded physical therapy. Intralesional steroid injections are given in the carpal tunnel and/or in the cervical region (transforaminal epidural/ translaminar/ selective nerve root block) if symptoms persist.

Surgical options for cervical radiculopathy include Anterior cervical discectomy and fusion (ACDF), cervical disc replacement or posterior decompression (laminoforaminotomy). Surgery for CTS involves open or endoscopic release of the transverse carpal ligament. The need for one or both surgeries and the order in which they should be performed should be individualized based on patient's symptoms and investigations. Suboptimal clinical response post carpal tunnel injection is poor prognostic indicator for carpal tunnel surgery performed alone in patients with CTS. ${ }^{3}$ A study by Baba et al suggests that cervical decompression should take precedence over the distal surgery to halt or prevent progression to myelopathy. ${ }^{11}$ Despite surgeries to relieve compression at both sites, the outcome still remains poor in patients with DCS. ${ }^{19}$

\section{CONTROVERSY}

Double crush implies mechanical compression at two focal points. It fails to take into consideration the multiple etiologies (endocrine, vascular, metabolic, inflammatory, infectious, genetic, nutritional, anatomical or idiopathic) causing nerve dysfunction. Upton and McComas themselves acknowledged the failure of the term they coined in cases where a nerve was affected at three or more sites. ${ }^{1}$ Poor results despite surgical decompressions at both sites further question the double compression hypothesis. ${ }^{19}$ Cohen et al have proposed to use a new term "multifocal neuropathy" instead of DCS to describe an interplay of complex processes causing neuronal dysfunction. ${ }^{20}$ Unfortunately, this term is already used for an autoimmune disease called multifocal motor neuropathy. 


\section{CONCLUSION}

Despite five decades of extensive research, the exact understanding of DCS still remains an unsolved puzzle. Further studies are warranted to establish the definitive risk factors, pathophysiology and an efficient treatment protocol. A clinician must always be suspicious of the possibility of DCS in patients presenting with CTS. A thorough physical examination aided by electrodiagnostic tests and imaging should be carried out to establish the diagnosis. In conjunction to relieving local compression, treatment must be directed at all the contributive systemic etiologies to achieve better results.

Funding: No funding sources

Conflict of interest: None declared

Ethical approval: Not required

\section{REFERENCES}

1. Upton AR, McComas AJ. The double crush in nerve entrapment syndromes. Lancet. 1973;2(7825):35962.

2. Massey EW, Riley TL, Pleet AB. Coexistent carpal tunnel syndrome and cervical radiculopathy (double crush syndrome). South Med J. 1981;74(8):957-59.

3. Osterman AL. The double crush syndrome. Orthop Clin North Am. 1988;19(1):147-55.

4. Morgan G, Wilbourn AJ. Cervical radiculopathy and coexisting distal entrapment neuropathies: doublecrush syndromes? Neurology. 1998;50(1):78-83.

5. Hurst LC, Weissberg D, Carroll RE. The relationship of the double rush to carpal tunnel syndrome (an analysis of 1,000 cases of carpal tunnel syndrome). J Hand Surg Br. 1985;10(2):202-04.

6. Aroori S, Spence RA. Carpal tunnel syndrome. Ulster Med J. 2008;77(1):6-17.

7. Atroshi I, Gummesson C, Johnsson R, Ornstein E, Ranstam J, Rosén I. Prevalence of carpal tunnel syndrome in a general population. JAMA. 1999;282(2):153-58.

8. Radhakrishnan K, Litchy WJ, O'Fallon WM, Kurland LT. Epidemiology of cervical radiculopathy. A population-based study from Rochester, Minnesota, 1976 through 1990. Brain. 1994;117(2):325-35.

9. Sang-Hyun C, Jae-Ho M, Soeng-Wung K. The Incidence of Median Nerve Double Crush Syndrome among the Carpal Tunnel Syndrome Patients. J K Acad Rehab Med. 1994;18(2):30.

10. Dorwart BB. Carpal tunnel syndrome: A review. Semin Arthritis Rheum. 1984;14(2):134-40.
11. Baba H, Maezawa Y, Uchida K. Cervical myeloradiculopathy with entrapment neuropathy: a study based on the double-crush concept. Spinal Cord. 1998;36(6):399-404.

12. Moghtaderi A, Izadi S. Double crush syndrome: an analysis of age, gender and body mass index. Clin Neurol Neurosurg. 2008;110(1):25-9.

13. Kane PM, Daniels AH, Akelman E. Double Crush Syndrome. J Am Acad Orthop Surg. 2015;23(9):55862.

14. Schmid AB, Coppieters MW. The double crush syndrome revisited - A Delphi study to reveal current expert views on mechanisms underlying dual nerve disorders. Man Ther. 2011;16(6):557-62.

15. Katz JN, Larson MG, Sabra A. Carpal tunnel syndrome diagnostic utility of history and physical examination findings. Ann Intern Med. 1990;112:321-27.

16. Jablecki CK, Andary MT, So YT, Wilkins DE, Williams FH. Literature review of the usefulness of nerve conduction studies and electromyography for the evaluation of patients with carpal tunnel syndrome. AAEM Quality Assurance Committee. Muscle Nerve. 1993;16(12):1392-414.

17. Bodofsky EB, Wu KD, Campellone JV, Greenberg WM, Tomaio AC. A sensitive new median-ulnar technique for diagnosing mild Carpal Tunnel Syndrome. Electromyogr Clin Neurophysiol. 2005;45(3):139-44.

18. Wang R, Liu Z, Wang W. Carpal tunnel syndrome with cervical spondylotic radiculopathy: a clinical and electrophysiological study. Zhonghua yi xue za zhi. 2015;95(35):2846-50.

19. Wessel LE, Fufa DT, Canham RB, La Bore A, Boyer MI, Calfee RP. Outcomes following Peripheral Nerve Decompression with and without Associated Double Crush Syndrome: A Case Control Study. Plast Reconstr Surg. 2017;139(1):119-27.

20. Cohen BH, Gaspar MP, Daniels AH, Akelman E, Kane PM. Multifocal Neuropathy: Expanding the Scope of Double Crush Syndrome. J Hand Surg Am. 2016;41(12):1171-75.

Cite this article as: Kuruwa DR, Elango E, Shah KA. Double crush syndrome: clinical review of an unsolved puzzle. Int J Res Orthop 2020;6:1362-4. 